\title{
Diffraction and near-zero transmission of flexural phonons at graphene grain boundaries
}

\author{
Edit E. Helgee and Andreas Isacsson* \\ Department of Applied Physics, Chalmers University of Technology, SE-412 96 Göteborg, Sweden \\ (Received 17 February 2015; revised manuscript received 24 April 2015; published 26 May 2015)
}

\begin{abstract}
Graphene grain boundaries are known to affect phonon transport and thermal conductivity, suggesting that they may be used to engineer the phononic properties of graphene. Here, the effect of two buckled grain boundaries on long-wavelength flexural acoustic phonons has been investigated as a function of angle of incidence using molecular dynamics. The flexural acoustic mode has been chosen due to its importance to thermal transport. It is found that the transmission through the boundaries is strongly suppressed for incidence angles close to $35^{\circ}$. Also, the grain boundaries are found to act as diffraction gratings for the phonons.
\end{abstract}

DOI: 10.1103/PhysRevB.91.205432

PACS number(s): $62.30 .+\mathrm{d}$, 68.60.Bs, 68.65.Pq

\section{INTRODUCTION}

Grain boundaries in graphene have been found to affect the mechanical, electronic, and thermal properties of the material [1-8]. The grain boundaries commonly consist of dislocations in the form of pentagon-heptagon defect pairs, and cause out-of-plane buckling of the graphene sheet [9-16]. Recent experimental studies show that dislocations can be introduced into pristine graphene using a focused electron beam [13,14,17-19], suggesting the possibility of adjusting the properties of the material.

The possibility of manipulating the properties of graphene could be particularly important in applications related to phononics and heat management [20,21], where control of the vibrational properties and thermal conductivity of graphene is essential. The effect of grain boundaries on the thermal conductivity of graphene has previously been studied using both molecular dynamics (MD) and Green's-function methods [22-28]. However, out of these studies only Liu et al. [28], who consider transport along the boundary, mention the influence of out-of-plane buckling. Also, these studies give no detailed insight into the scattering processes of specific phonon modes.

In the present study, we investigate the scattering of long-wavelength flexural acoustic phonons at grain boundaries in graphene for several incidence angles using molecular dynamics. This particular phonon mode was chosen since it is believed to contribute significantly to the thermal conductivity [29,30]. Two grain boundaries are considered in this paper, one with a misorientation angle of $9.4^{\circ}$ and one with a misorientation angle of $17.9^{\circ}$. Both grain boundaries display substantial out-of-plane buckling, with a periodic variation in height along the grain boundary due to the distribution of defects. The boundaries are found to act as diffraction gratings for the phonons, and strongly suppressed transmission is also observed for specific angles. In particular, the transmission is as low as $4 \%$ for incidence angles near $35^{\circ}$ at both boundaries.

A previous investigation limited to phonons normally incident on the grain boundary showed that the scattering was due almost entirely to the out-of-plane buckling of the boundary [31]. Based on this result a continuum mechanical model was constructed, where the grain boundary was modeled

\footnotetext{
*andreas.isacsson@chalmers.se
}

as a static out-of-plane displacement. The model showed good agreement with the MD results. Here, we extend this continuum mechanical model to the case of non-normal angle of incidence in order to gain a qualitative understanding of the scattering mechanism.

\section{METHOD}

All MD simulations have been performed using the program package LAMMPS (large-scale atomic/molecular massively parallel simulator) [32]. The interaction between carbon atoms has been modeled using the Tersoff potential [33,34] with the potential parameters given by Lindsay and Broido [35]. This set of parameters has been chosen due to its improved description of acoustic phonon modes in graphene. The considered grain boundaries are symmetric tilt grain boundaries and consist of periodic arrays of pentagon-heptagon defects. The $9.4^{\circ}$ grain boundary has a period of $1.5 \mathrm{~nm}$ in the $y$ direction, parallel to the grain boundary (see Fig. 1), while the $17.9^{\circ}$ boundary shown in Fig. 2 has a period of $2.4 \mathrm{~nm}$. The grain boundaries have been constructed using the method described in Ref. [31]. For the $9.4^{\circ}$ boundary this results in a grain boundary buckling $0.6 \mathrm{~nm}$ high and $1.7 \mathrm{~nm}$ wide. Due to the defect distribution the buckling height varies periodically along the grain boundary with an amplitude of $0.06 \mathrm{~nm}$. The $17.9^{\circ}$ boundary has a buckling height of $1.5 \mathrm{~nm}$ and a buckling width of $5 \mathrm{~nm}$, with a variation of $0.1 \mathrm{~nm}$ along the boundary.

In the investigation of phonon scattering, the phonons introduced into the system must have a well-defined wave vector and polarization. Also, the finite size of the simulation supercell makes it necessary to use localized phonon wave packets rather than plane waves. To construct phonon wave packets with the required properties we use the method of Kimmer et al. [37]. The displacement $\mathbf{u}_{j}$ of atom $j$ is then determined by

$$
\mathbf{u}_{j}=\operatorname{Re}\left[\sum_{\mathbf{k}} a_{\mathbf{k}} \vec{\epsilon}_{j \mathbf{k}} e^{i \mathbf{k} \cdot \mathbf{R}_{j}-i \omega(\mathbf{k}) t}\right],
$$

where $\mathbf{k}=k_{x} \hat{x}+k_{y} \hat{y}$ is a wave vector, $\vec{\epsilon}_{j \mathbf{k}}$ is a polarization vector for the considered phonon branch, $\mathbf{R}_{j}$ is the position vector of atom $j$, and $\omega$ is the phonon frequency. The amplitudes $a_{\mathbf{k}}$ are calculated according to

$$
a_{\mathbf{k}}=A e^{-\eta^{2}\left(k_{x}-k_{0 x}\right)^{2}} e^{-i \mathbf{k} \cdot \mathbf{R}_{0}},
$$




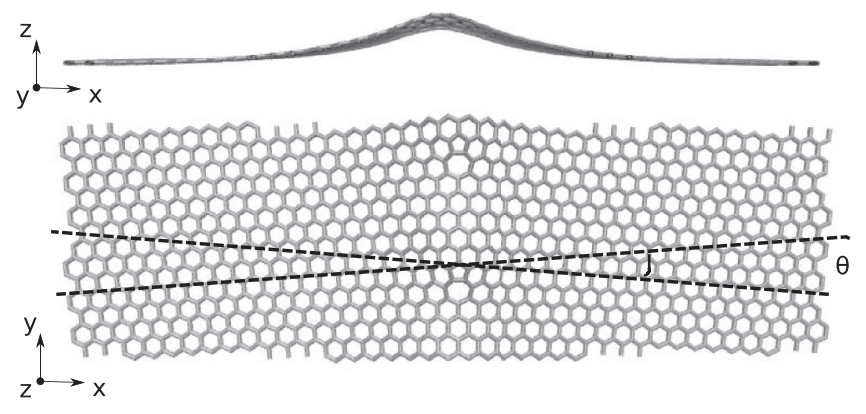

FIG. 1. Symmetric tilt grain boundary with misorientation angle $9.4^{\circ}$, seen from the $y$ direction (top) and $z$ direction (bottom). Figure made using VMD [36].

where $A$ is an amplitude and $\eta$ is the width of the wave packet in the $x$ direction (perpendicular to the grain boundary). The functional form of the amplitude is chosen so that the resulting wave packet has a Gaussian shape and is localized in the $x$ direction, centered around $\mathbf{R}_{0}$ in real space and around a wave vector $\mathbf{k}_{0}=k_{x 0} \hat{x}+k_{y} \hat{y}$ in reciprocal space. All wave vectors $\mathbf{k}$ are required to be reciprocal lattice vectors of the simulation supercell. With periodic boundary conditions applied in the $y$ direction (parallel to the grain boundary) this gives $k_{y}=$ $2 \pi m / L_{y}^{\text {cell }}$, where $m$ is an integer and $L_{y}^{\text {cell }}$ is the size of the supercell in the $y$ direction.

The polarization vectors $\vec{\epsilon}_{j \mathbf{k}}$ and dispersion relation $\omega(\mathbf{k})$ have been obtained from the dynamical matrix of the perfect lattice using the General Utility Lattice Program, GULP $[38,39]$. The constant $A$ has been set to the rather small value 0.013 $\mathrm{nm}$ in order to avoid the nonlinear effects observed for high-amplitude vibrations in graphene. For the width $\eta$ a value of $5 \mathrm{~nm}$ has been chosen in order to make the wave packet fairly broad in real space and narrow in reciprocal space. This is important as the quadratic dispersion of the flexural mode would otherwise cause the wave packet to become very distorted over time.

Since the focus of this study is long-wavelength phonons, the upper limit for $\left|\mathbf{k}_{0}\right|=\sqrt{k_{x 0}^{2}+k_{y}^{2}}$ has been set to $7 \mathrm{~nm}^{-1}$, which limits the possible values of $m$ and $k_{x 0}$. To extend the range of possible $m$ values the size of the simulation supercells in the $y$ direction has been increased relative to the grain boundary periodicity. For the $9.4^{\circ}$ boundary it has been tripled, so that $L_{y}^{\text {cell }}=4.5 \mathrm{~nm}$, while for the $17.9^{\circ}$ boundary it has been doubled, giving $L_{y}^{\text {cell }}=4.8 \mathrm{~nm}$. The supercells of the 9.4 and $17.9^{\circ}$ boundaries are 260 and $400 \mathrm{~nm}$ long in the $x$ direction, respectively. Fixed boundary conditions are applied in this direction and all atoms less than $10 \mathrm{~nm}$ from the supercell edge are held immobile. Note that there is no temperature in these simulations, and the wave packet therefore does not interact with any thermally excited phonons, but only with the static grain boundary buckling.

In our previous study of phonon scattering at graphene grain boundaries a simple continuum mechanical model of the system was constructed in order to further confirm the results and to facilitate future studies of systems too large to model using MD [31]. The model built on the observation that the main cause for scattering of long-wavelength phonons at the grain boundary is the buckling. Here, we have extended the previously used model from one to two dimensions for the case of the $9.4^{\circ}$ boundary, and incorporated the periodic height variation of the buckling.

The equations of motion for the displacements are

$$
\begin{gathered}
\rho \ddot{u}-\partial_{x} \sigma_{x x}-\partial_{y} \sigma_{x y}=0, \\
\rho \ddot{v}-\partial_{x} \sigma_{x y}-\partial_{y} \sigma_{y y}=0, \\
\rho \ddot{w}+\kappa \Delta^{2} w-\partial_{x}\left[\sigma_{x x} \partial_{x} w+\sigma_{x y} \partial_{y} w\right] \\
-\partial_{y}\left[\sigma_{x y} \partial_{x} w+\sigma_{y y} \partial_{y} w\right]=0,
\end{gathered}
$$

where $u$ is the displacement in $x ; v$ is the displacement in $y$; $w$ is the out-of-plane displacement; $\rho$ is the density; $\kappa$ is the bending rigidity; and $\sigma_{x x}, \sigma_{x y}$, and $\sigma_{y y}$ are the components of the stress tensor. As in the previous study the grain boundary buckling has been included in the form of a static out-of-plane displacement.

Finite-difference methods have been used to propagate wave packets similar to the ones used in MD and to study scattering against the buckling. Results of these calculations can be directly compared to the MD simulation results. The details of the continuum mechanical model can be found in the Appendix.

\section{RESULTS}

The time evolution of the kinetic energy in both grains for a wave packet with $k_{x 0}=4 \mathrm{~nm}$ and $m=2$ interacting with the $9.4^{\circ}$ boundary can be seen in Fig. 3. Here, grain 1 is defined as the grain in which the pulse is introduced, and grain 2 is the other grain. Changes in the kinetic energy of the grains

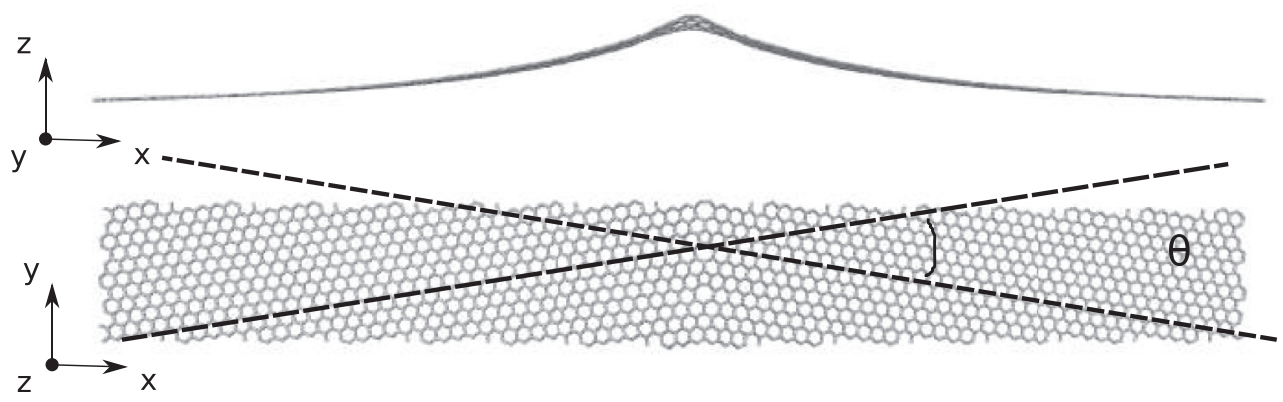

FIG. 2. Symmetric tilt grain boundary with misorientation angle $17.9^{\circ}$, seen from the $y$ direction (top) and $z$ direction (bottom). Figure made using VMD [36]. 


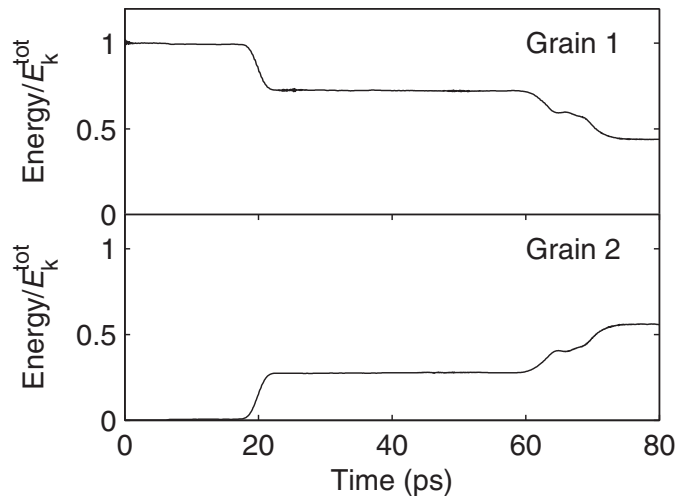

FIG. 3. The fraction of the total kinetic energy in grain 1 (top) and grain 2 (bottom) as a function of time for a wave packet with $k_{x 0}=4 \mathrm{~nm}^{-1}$ and $m=2$ scattering at the $9.4^{\circ}$ boundary.

can be seen at two points. After 20 ps, the kinetic energy in grain 1 decreases to $73 \%$ of the total kinetic energy while the kinetic energy of grain 2 increases to $27 \%$, indicating that the pulse has reached the grain boundary. The second change occurs at $60 \mathrm{ps}$, where the energy of grain 1 decreases further in two steps, first to $60 \%$ and then to $44 \%$. Between these two points the pulse has been reflected against the fixed boundary conditions, so that the steps at $60 \mathrm{ps}$ mark the return of the scattered pulses to the grain boundary.

The most surprising feature of Fig. 3 is the stepwise change in energy beginning at $60 \mathrm{ps}$, which seems to indicate that there are two pulses arriving at the grain boundary about 5 ps apart. A closer examination of the scattered pulses shows that this is indeed the case. Figure 4 shows the intensity of the scattered pulses, normalized by the total intensity, as a function of wave vectors $k_{x}$ and $k_{y}$ for $t=40 \mathrm{ps}$. Four peaks are seen, two with negative $k_{x}$, corresponding to reflected pulses, and two transmitted pulses with positive $k_{x}$. The reflected

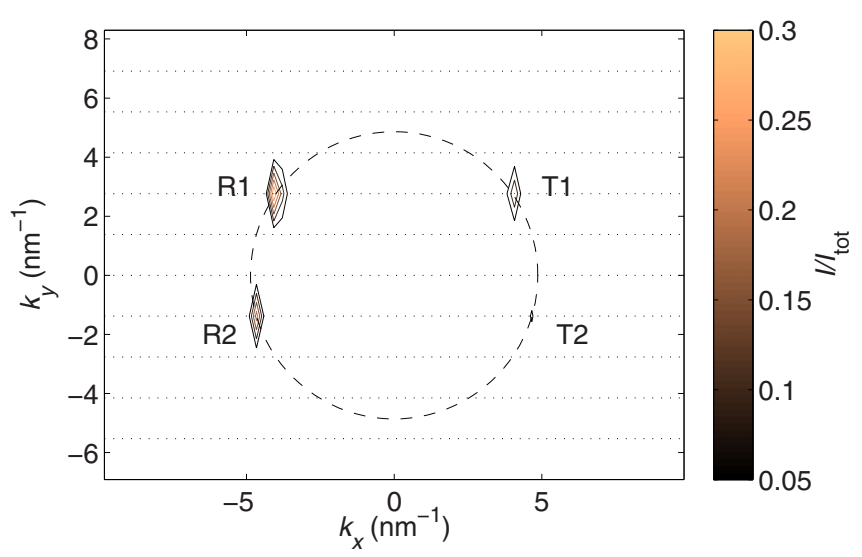

FIG. 4. (Color online) Normalized intensity $I / I_{\text {tot }}$ after scattering at the $9.4^{\circ}$ boundary $(t=40 \mathrm{ps})$ as a function of $k_{x}$ and $k_{y}$ for a wave packet with $k_{x 0}=4 \mathrm{~nm}^{-1}$ and $m=2$. R1 and R2 denote the reflected pulses, while $\mathrm{T} 1$ and $\mathrm{T} 2$ are the transmitted pulses. The dotted lines represent the values of $k_{y}$ allowed by the boundary conditions, and the dashed circle indicates the points with $k_{0}=\sqrt{k_{x}^{2}+k_{y}^{2}}$ equal to that of the incident pulse.

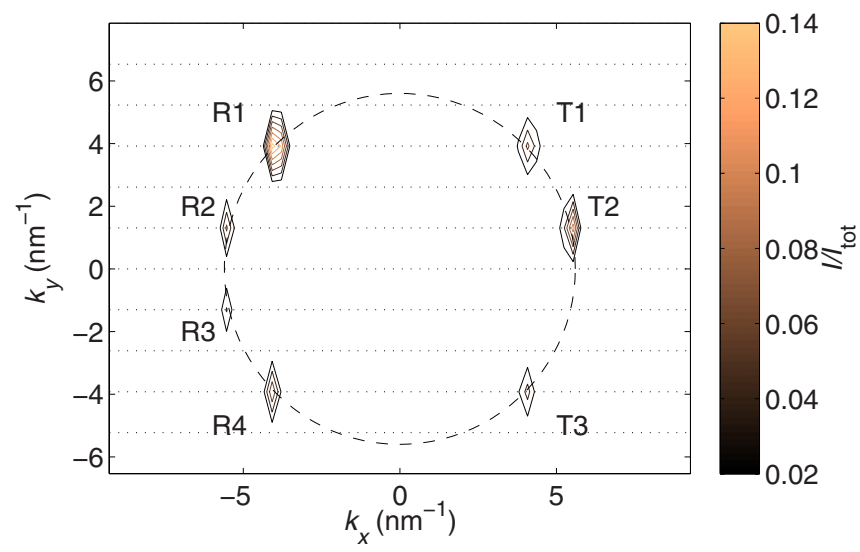

FIG. 5. (Color online) Normalized intensity $I / I_{\text {tot }}$ after scattering at the $17.9^{\circ}$ grain boundary as a function of $k_{x}$ and $k_{y}$ for a wave packet with $k_{x 0}=4 \mathrm{~nm}^{-1}$ and $m=3$. R1 to R4 denote the reflected pulses while T1, T2, and T3 are the transmitted pulses. The dotted lines represent the values of $k_{y}$ allowed by the boundary conditions, and the dashed circle indicates the points with $k_{0}=\sqrt{k_{x}^{2}+k_{y}^{2}}$ equal to that of the incident pulse.

pulses are labeled R1 and R2. R1 has $k_{x}=-4 \mathrm{~nm}^{-1}$ and $k_{y}=2.8 \mathrm{~nm}^{-1}$, while R2 occurs at $k_{x}=-4.7 \mathrm{~nm}^{-1}$ and $k_{y}=-1.4 \mathrm{~nm}^{-1}$. Similarly, the transmitted pulses T1 and T2 have $k_{x}=4, k_{y}=2.8 \mathrm{~nm}^{-1}$ and $k_{x}=4.7, k_{y}=-1.4 \mathrm{~nm}^{-1}$, respectively. T1 has the same wave vector as the incident pulse. Since the propagation velocity of the pulse depends on the value of $k_{x}$, the pulses will propagate with different velocities and thus give rise to the stepwise change in kinetic energy seen in Fig. 3.

The same phenomenon is observed at the $17.9^{\circ}$ grain boundary. Figure 5 shows the normalized intensity after scattering for a pulse with $k_{x 0}=4 \mathrm{~nm}^{-1}$ and $m=3$. Four reflected peaks and three transmitted peaks can be seen. For the reflected peaks, $\mathrm{R} 1$ occurs at $k_{x}=-4.0, k_{y}=3.9 ; \mathrm{R} 2$ occurs at $k_{x}=-5.5, k_{y}=1.3$; R3 occurs at $k_{x}=-5.5, k_{y}=$ -1.3 ; and R4 occurs at $k_{x}=-4.0, k_{y}=-3.9 \mathrm{~nm}^{-1}$, while the transmitted peaks occur at $k_{x}=4.0, k_{y}=3.9$ (T1); $k_{x}=$ $5.5, k_{y}=1.3(\mathrm{~T} 2)$; and $k_{x}=4.0, k_{y}=-3.9 \mathrm{~nm}^{-1}$ (T3).

Examination of the scattered pulses at both grain boundaries reveals that the difference between the $k_{y}$ value for the incident pulse, $k_{y}^{\text {in }}$, and the $k_{y}$ value for the scattered pulses, $k_{y}^{\text {sc }}$, can be expressed as

$$
k_{y}^{\mathrm{sc}}-k_{y}^{\text {in }}=\frac{2 \pi n}{L_{y}},
$$

where $n$ is an integer and $L_{y}$ is the grain boundary period. The $k_{x}$ value of the scattered pulses, $k_{x}^{\text {sc }}$, is given by momentum conservation:

$$
k_{x}^{\mathrm{sc}}=\sqrt{\left(k_{0}^{\mathrm{in}}\right)^{2}-\left(k_{y}^{\mathrm{sc}}\right)^{2}} .
$$

This indicates that the buckled, periodic grain boundaries act as diffraction gratings for long-wavelength flexural acoustic phonons.

It should be mentioned that for the $9.4^{\circ}$ grain boundary a larger simulation supercell has been used to verify that the above result is not a consequence of the periodic boundary 
conditions or limited supercell size. The larger supercell had a size of $6 \mathrm{~nm}$ in the $y$ direction, corresponding to four grain boundary periods. If the periodic boundary conditions had been the cause of the diffraction, this larger supercell would be expected to produce a different result compared to the smaller supercell. In particular, diffraction due to the periodic boundary conditions should have created pulses with $k_{y}^{\text {sc }}$ determined by

$$
k_{y}^{\mathrm{sc}}-k_{y}^{\mathrm{in}}=\frac{2 \pi n}{L_{y}^{\mathrm{cell}}},
$$

where $L_{y}^{\text {cell }}$ is the supercell width in the $y$ direction. Since $L_{y}^{\text {cell }}=4 L_{y}$ for the larger supercell, this would produce a change in the $y$ component of the wave vector that is four times smaller than the one given by Eq. (6). This change should be observable for all values of $m$ and $k_{x 0}$. Instead, the larger supercell yields the same results as the smaller supercell with regard to both total transmission and diffraction, and the difference in $k_{y}$ between the incoming and scattered pulses is found to depend on the inherent grain boundary periodicity as in Eq. (6). This shows that it is the inherent grain boundary period $L_{y}$, and not the supercell size $L_{y}^{\text {cell }}$, that plays a part in the diffraction. Grain boundaries functioning as diffraction gratings for phonons have previously been used to model the behavior of the thermal conductivity in ionic materials [40].

Unlike the previously described case where $m=0$ [31], scattering into in-plane vibrational modes is negligible for all cases with $m=1$ and 2, while some movement in the $y$ direction is seen at the $9.4^{\circ}$ boundary for $m=3$. This difference is due to the restrictions on $k_{y}$ imposed by the periodic boundary conditions. The in-plane vibrations excited at the grain boundary will have the same frequency as the incoming flexural vibrations, but due to the differences in the dispersion relation this corresponds to a much smaller wave vector, and thus a longer wavelength, for the in-plane modes. If there is a substantial $y$ component in the wave vector, the in-plane vibration will thus not fit into the simulation supercell. In practice, this means that only in-plane modes with $k_{y}=0$ can propagate in the present simulation setup. Thus, vibrations in the longitudinal mode can be engendered by normally incident pulses as previously reported [31], and transverse vibrations may be excited at high incidence angles as seen in the present study. If it had been possible to include in-plane vibrations with nonzero $k_{y}$ the obtained results would most likely be qualitatively equivalent to the results presented here, although some quantitative differences could be expected.

The transmission coefficient $T$ is defined as

$$
T=\frac{\left\langle E_{\mathrm{k}}^{\text {grain2}}\right\rangle}{E_{\mathrm{k}}^{\mathrm{tot}}},
$$

where $E_{\mathrm{k}}^{\text {grain2 }}$ is the kinetic energy in grain $2, E_{\mathrm{k}}^{\mathrm{tot}}$ is the total kinetic energy, and the brackets represent a time average over times between the first scattering at the grain boundary and the time when the first wave packets reaches the edge of the supercell. This is the total transmission coefficient, including contributions from both in-plane and flexural modes. Values of $T$ for the $9.4^{\circ}$ grain boundary for several values of $k_{x 0}$ at $m=1,2$, and 3 can be seen in Fig. 6. For all values of $m$, the transmission increases with increasing $k_{x 0}$. The increase

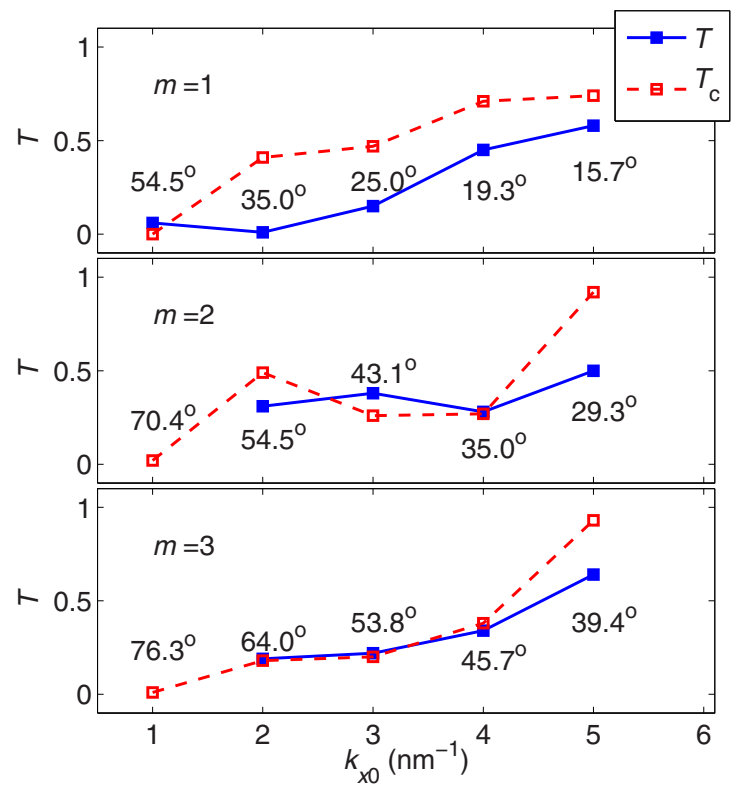

FIG. 6. (Color online) Transmission at the $9.4^{\circ}$ boundary as a function of $k_{x 0}$ for $m=1$ (top), $m=2$ (middle), and $m=3$ (bottom). The angle of incidence is indicated beside each data point. Open symbols and dashed lines represent results from the continuum mechanical model.

is monotonic for $m=3$, while for $m=2$ there is a small dip around $k_{x 0}=4 \mathrm{~nm}^{-1}$ and for $m=1$ there is a pronounced trough around $k_{x 0}=2 \mathrm{~nm}^{-1}$. Remarkably, the transmission for $m=1$ and $k_{x 0}=2 \mathrm{~nm}^{-1}$ nearly reaches zero, so that no part of the incident pulse is transmitted through the boundary. It can be noted that the dips in the curve for $m=2$ and the trough for $m=1$ occur at the same angle $35^{\circ}$, but for different values of $k_{0}$.

Figure 7 shows the dependence of $T$ on $k_{x 0}$ with $m=1$, 2 , and 3 for the $17.9^{\circ}$ boundary. As for the $9.4^{\circ}$ boundary, the transmission increases with increasing $k_{x 0}$. Extremely low transmission is also observed at $m=1$ and $k_{x 0}=2 \mathrm{~nm}^{-1}$, corresponding to an incidence angle of $33^{\circ}$. It is not clear whether there is a minimum at the same angle of incidence for $m=2$, as in the $9.4^{\circ}$ case, as the transmission is quite low also at slightly larger incidence angles.

Figure 6 also contains transmission coefficients $T_{\mathrm{c}}$ obtained from the continuum mechanical model. The qualitative agreement between the continuum mechanical model and the MD results is very good, as the continuum mechanical model clearly reproduces the general trend in the MD data of increasing transmission with increasing $k_{x 0}$. The two models agree particularly well for $m=3$, although the continuum mechanical model overestimates the transmission at $k_{x 0}=$ $5 \mathrm{~nm}^{-1}$. For $m=2$, the dip around $k_{x 0}=4 \mathrm{~nm}^{-1}$ is reproduced but is wider than in the MD data, extending to $k_{x 0}=3 \mathrm{~nm}^{-1}$. The continuum mechanical model also overestimates the transmission at $k_{x 0}=5 \mathrm{~nm}^{-1}$. Finally, for $m=1$ the transmission obtained with the continuum mechanical model is higher than that obtained with MD over almost the entire interval. It also does not reproduce the trough at $k_{x 0}=2 \mathrm{~nm}^{-1}$, but does reach near-zero values for $k_{x 0}=1 \mathrm{~nm}^{-1}$. 


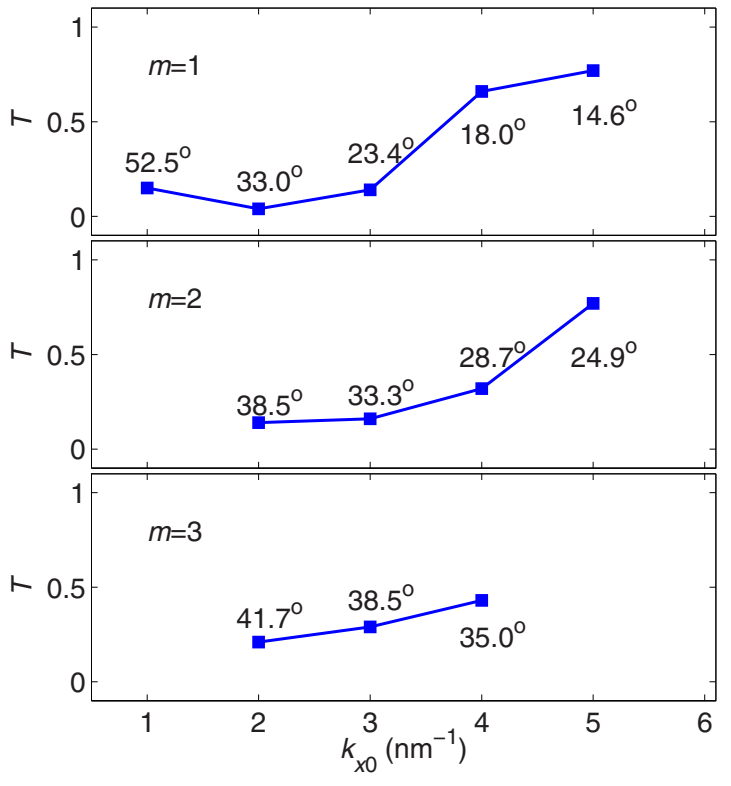

FIG. 7. (Color online) Transmission at the $17.9^{\circ}$ boundary as a function of $k_{x 0}$ for $m=1$ (top), $m=2$ (middle), and $m=3$ (bottom). The angle of incidence is indicated beside each data point.

The lack of quantitative agreement that is seen especially for $m=1$ is most likely due to differences between the buckling obtained in MD simulations and that used in the continuum mechanical model. As can be seen in the Appendix, the buckling in the continuum mechanical model is described using a minimum model with only four adjustable parameters. This description has the great advantage of being simple, but it is not able to correctly reproduce every feature of the buckling obtained from MD simulations. Hence, some quantitative disagreement between MD and the continuum mechanical model is to be expected. That the discrepancy is especially large for smaller values of $m$ may be a consequence of the fact that wave packets with different values of $m$ interact with different features of the boundary. The features important

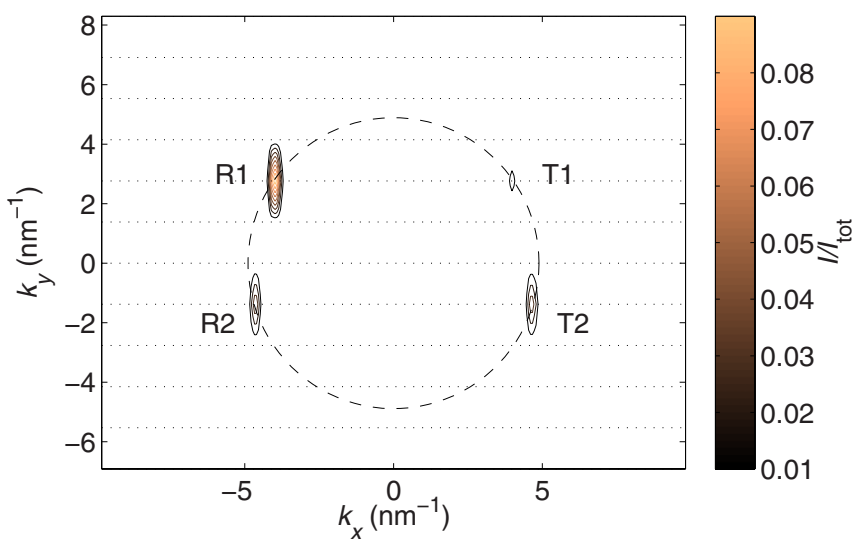

FIG. 8. (Color online) Normalized intensity after scattering as a function of $k_{x}$ and $k_{y}$ for a wave packet with $k_{x 0}=4 \mathrm{~nm}^{-1}$ and $m=2$, from the continuum mechanical model. The dotted lines represent the values of $k_{y}$ allowed by the boundary conditions, and the dashed circle indicates the points with $k_{0}=\sqrt{k_{x}^{2}+k_{y}^{2}}$ equal to that of the incident pulse. for scattering of wave packets with $m=1$ and 2 are thus not necessarily as accurately described by the present model as those affecting wave packets with $m=3$.

In addition to the transmission coefficient, the continuum mechanical model should reproduce the diffraction seen in MD. Figure 8 shows the intensity obtained from the continuum mechanical model after scattering as a function of $k_{x}$ and $k_{y}$ for $k_{x 0}=4 \mathrm{~nm}^{-1}$ and $m=2$, corresponding to the MD results presented in Fig. 4. It is clear that the same peaks appear, showing that diffraction occurs also in the continuum mechanical model. Compared to the MD results T1 appears to be underestimated and T2 appears to be overestimated, possibly because the model of the boundary buckling used in the continuum mechanical model does not reproduce the actual curvature of the grain boundary buckling in sufficient detail.

\section{CONCLUSION}

In summary, the effects of the angle of incidence on the scattering of long-wavelength flexural phonons against grain boundaries in graphene have been studied using molecular dynamics. The considered grain boundaries, two buckled symmetric tilt grain boundaries with misorientation angles 9.4 and $17.9^{\circ}$, have been found to act as diffraction gratings for long-wavelength flexural phonons. In addition, near-zero transmission has been observed for angles near $35^{\circ}$ and small wave-vector magnitudes. A continuum mechanical model of the system containing the $9.4^{\circ}$ boundary has been constructed and shown to qualitatively agree with the MD results, giving insights into the scattering mechanism and providing a starting point for studies of systems too large to be modeled atomistically. The presented results improve our understanding of how phonons interact with grain boundaries in graphene and suggest that such defects could indeed be useful in manipulating the vibrational properties of the material.

\section{ACKNOWLEDGMENTS}

The authors would like to thank Prof. Jari Kinaret for rewarding discussions. We also acknowledge financial support from the Swedish Research Council (VR) and the European Union Graphene Flagship (Grant No. 604391).

\section{APPENDIX: CONTINUUM MECHANICAL MODELING}

In the continuum mechanical model the graphene sheet is described as a thin plate. The equations of motion for the displacements are

$$
\begin{gathered}
\rho \ddot{u}-\partial_{x} \sigma_{x x}-\partial_{y} \sigma_{x y}=0, \\
\rho \ddot{v}-\partial_{x} \sigma_{x y}-\partial_{y} \sigma_{y y}=0, \\
\rho \ddot{w}+\kappa \Delta^{2} w-\partial_{x}\left[\sigma_{x x} \partial_{x} w+\sigma_{x y} \partial_{y} w\right] \\
-\partial_{y}\left[\sigma_{x y} \partial_{x} w+\sigma_{y y} \partial_{y} w\right]=0,
\end{gathered}
$$

where $u$ is the displacement in $x$ (perpendicular to the boundary); $v$ is the displacement in $y$ (parallel to the boundary); $w$ is the out-of-plane displacement; $\kappa$ is the bending rigidity; $\rho$ is the density; and $\sigma_{x x}, \sigma_{x y}$, and $\sigma_{y y}$ are the elements of the two-dimensional stress tensor. To model the 
grain boundary buckling, a static out-of-plane displacement $w_{0}(x, y)$ is introduced. The introduction of this out-of-plane displacement gives rise to static displacements in the in-plane directions, so that the total displacements must be written

$$
\begin{gathered}
u(x, y, t)=u_{0}(x, y)+u_{1}(x, y, t), \\
v(x, y, t)=v_{0}(x, y)+v_{1}(x, y, t), \\
w(x, y, t)=w_{0}(x, y)+w_{1}(x, y, t),
\end{gathered}
$$

where $u_{1}(x, y, t), v_{1}(x, y, t)$ and $w_{1}(x, y, t)$ are the timedependent displacements. However, the displacements determine the stress tensor components through the relations

$$
\begin{aligned}
& \sigma_{x x}=(\lambda+2 \mu)\left[\partial_{x} u+\frac{\left(\partial_{x} w\right)^{2}}{2}\right]+\lambda\left[\partial_{y} v+\frac{\left(\partial_{y} w\right)^{2}}{2}\right], \\
& \sigma_{y y}=\lambda\left[\partial_{x} u+\frac{\left(\partial_{x} w\right)^{2}}{2}\right]+(\lambda+2 \mu)\left[\partial_{y} v+\frac{\left(\partial_{y} w\right)^{2}}{2}\right], \\
& \sigma_{x y}=\mu\left[\partial_{x} v+\partial_{y} u+\partial_{x} w \partial_{y} w\right],
\end{aligned}
$$

where $\lambda$ and $\mu$ are Lamé parameters. Thus, the stress tensor elements can also be divided into a time-dependent term $\sigma_{i j}^{1}(i, j=x, y)$ and a time-independent term $\sigma_{i j}^{0}$. The twodimensional stress tensor components $\sigma_{x x}^{0}, \sigma_{y y}^{0}$, and $\sigma_{x y}^{0}$ are related through the Airy stress function [41] $\chi$, such that

$$
\sigma_{x x}^{0}=\frac{\partial^{2} \chi}{\partial y^{2}}, \quad \sigma_{y y}^{0}=\frac{\partial^{2} \chi}{\partial x^{2}}, \quad \sigma_{x y}^{0}=-\frac{\partial^{2} \chi}{\partial x \partial y} .
$$

It follows that the time-independent terms of the stress tensor will vanish in Eqs. (A1) and (A2), but not in Eq. (A3). The equations of motion for the time-dependent displacements thus become

$$
\begin{aligned}
& \rho \ddot{u}_{1}-\partial_{x} \sigma_{x x}^{1}-\partial_{y} \sigma_{x y}^{1}=0, \\
& \rho \ddot{v}_{1}-\partial_{x} \sigma_{x y}^{1}-\partial_{y} \sigma_{y y}^{1}=0,
\end{aligned}
$$

$$
\begin{aligned}
& \rho \ddot{w}+\kappa \Delta^{2}\left(w_{0}+w_{1}\right) \\
& \quad-\partial_{x}\left[\left(\sigma_{x x}^{0}+\sigma_{x x}^{1}\right) \partial_{x}\left(w_{0}+w_{1}\right)+\left(\sigma_{x y}^{0}+\sigma_{x y}^{1}\right) \partial_{y}\left(w_{0}+w_{1}\right)\right] \\
& \quad-\partial_{y}\left[\left(\sigma_{x y}^{0}+\sigma_{x y}^{1}\right) \partial_{x}\left(w_{0}+w_{1}\right)+\left(\sigma_{y y}^{0}+\sigma_{y y}^{1}\right) \partial_{y}\left(w_{0}+w_{1}\right)\right] \\
& =0 .
\end{aligned}
$$

When solving these equations, any terms that are not linear in the derivatives of $u_{1}(x, t), v_{1}(x, t)$, or $w_{1}(x, t)$ can be ignored due to small vibrational amplitudes.

Finite-difference methods have been used to solve Eqs. (A1)-(A3). As in our previous paper [31], the equations have been discretized using standard discretization schemes [42] with step sizes $\Delta x=\Delta y=0.05 \mathrm{~nm}$ and $\Delta t=$ $0.4 \sqrt{d x^{4} / 4 \kappa}=0.8 \mathrm{fs}$. The Lamé parameters, bending rigidity, and density have been set to the values given by the modified Tersoff potential, i.e., $\mu=167 \mathrm{~N} \mathrm{~m}^{-1}, \lambda=23 \mathrm{~N} \mathrm{~m}^{-1}, \kappa=$ $2.8 \times 10^{-19} \mathrm{~J}$, and $\rho=7.42 \times 10^{-7} \mathrm{~kg} \mathrm{~m}^{-2}$. Fixed boundary conditions are applied in the $x$ direction and periodic boundary conditions are applied in the $y$ direction. The initial conditions are

$$
\begin{aligned}
w_{1}(x, y, t=0) & =\operatorname{Re}\left[\sum_{k} a_{\mathbf{k}} e^{i\left(\mathbf{k} \cdot \mathbf{R}-\omega\left(k_{0}\right) t\right)}\right], \\
\partial_{t} w_{1}(x, y, t=0) & =\operatorname{Re}\left[-i \omega\left(k_{0}\right) \sum_{k} a_{\mathbf{k}} e^{i\left(\mathbf{k} \cdot \mathbf{R}-i \omega\left(k_{0}\right) t\right)}\right],
\end{aligned}
$$

with

$$
a_{\mathbf{k}}=A e^{-\eta^{2}\left(k_{x}-k_{0 x}\right)^{2}} e^{-i \mathbf{k} \cdot \mathbf{R}_{0}} .
$$

As in the MD simulations, $\mathbf{k}=k_{x} \hat{x}+k_{y} \hat{y}$ is a wave vector allowed by the boundary conditions, $\mathbf{R}=x \hat{x}+y \hat{y}$ is a position, $A=0.01 \mathrm{~nm}$ is an amplitude, and $\eta=2 \mathrm{~nm}$ is the width of the wave packet. The wave packet is centered around

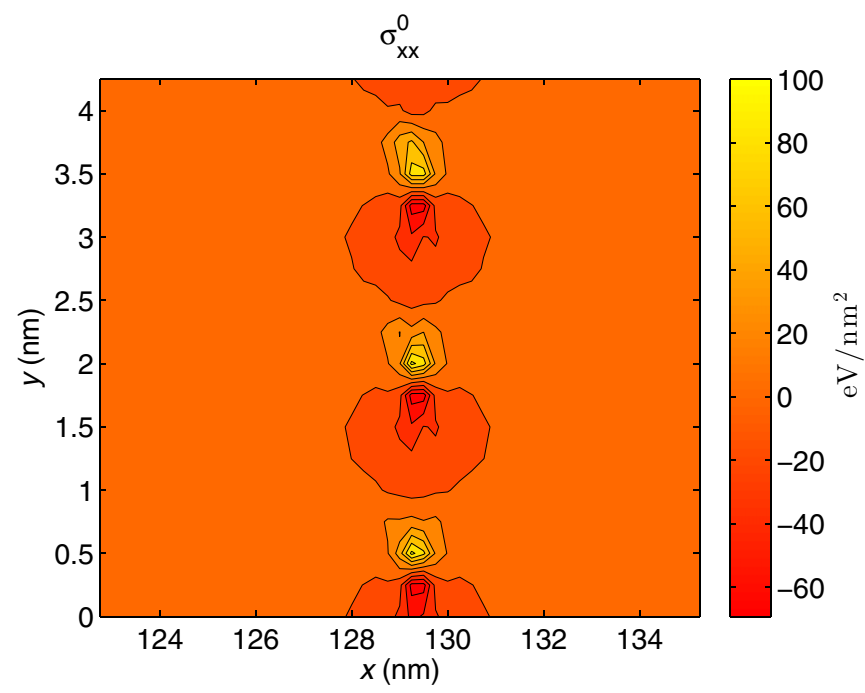

(a)

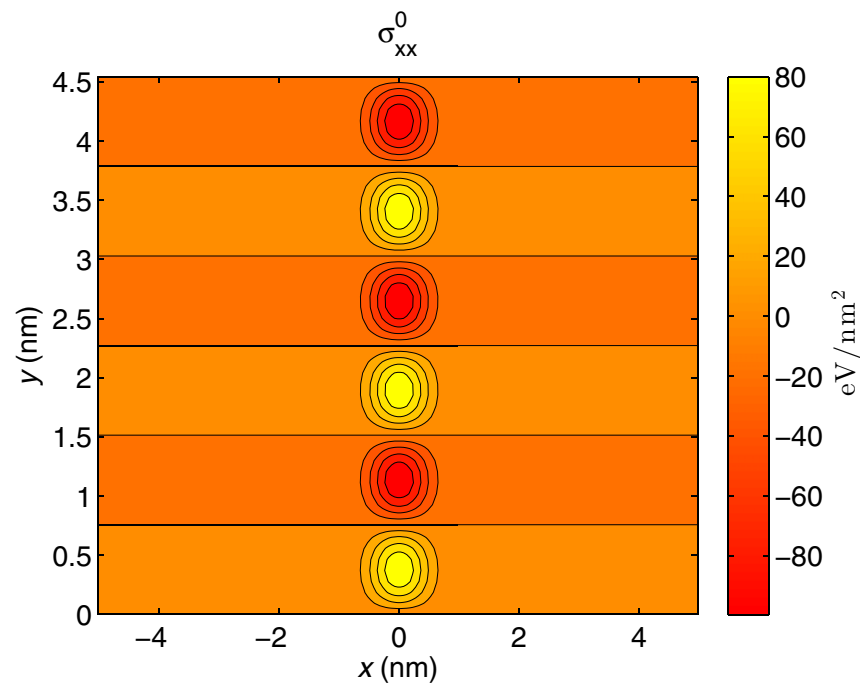

(b)

FIG. 9. (Color online) The stress tensor component $\sigma_{x x}^{0}$ close to the grain boundary (a) as obtained from MD and (b) as approximated according to Eq. (A15). Note that the grain boundary is located at $x=129 \mathrm{~nm}$ in the MD simulations and at $x=0$ in the continuum mechanical model. 


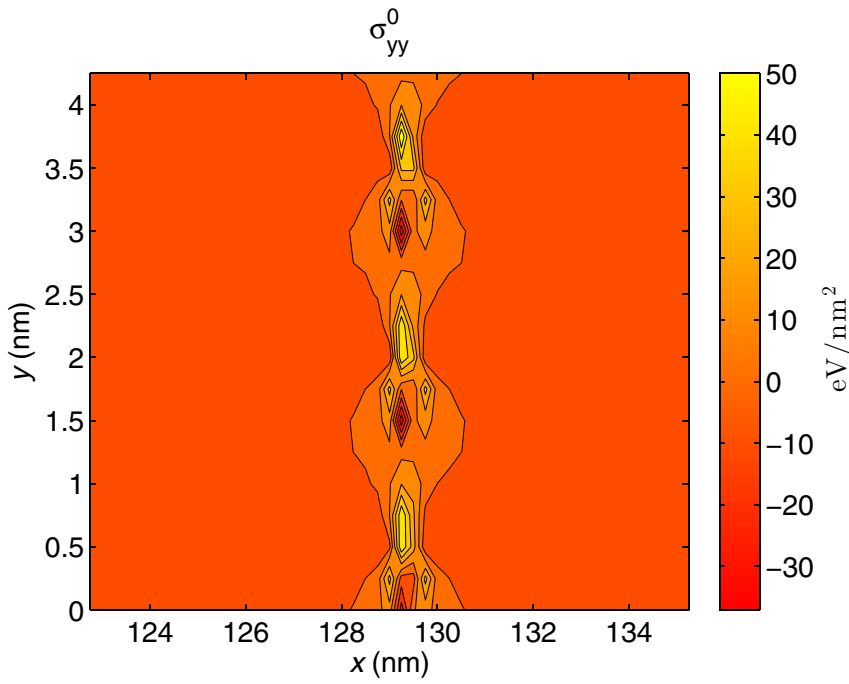

(a)

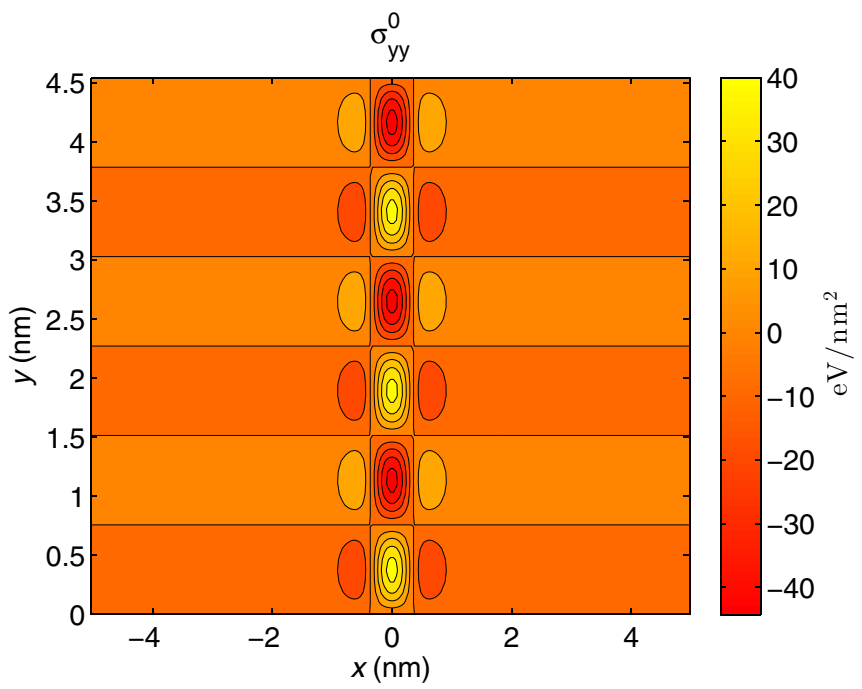

(b)

FIG. 10. (Color online) The stress tensor component $\sigma_{y y}^{0}$ (a) as obtained from MD and (b) as approximated according to Eq. (A16).

$\mathbf{R}_{0}$ in real space and $\mathbf{k}_{\mathbf{0}}=k_{x 0} \hat{x}+k_{y} \hat{y}$ in reciprocal space, and $\omega\left(k_{0}\right)$ is the frequency of out-of-plane vibrations with wave vector $k_{0}$.

The static out-of-plane displacement is set to

$$
w_{0}(x, y)=A_{\mathrm{b}} e^{-x^{2} / 2 \xi^{2}}\left[1+a \sin \left(\frac{2 \pi m y}{L_{y}}\right)\right],
$$

where $L_{y}$ is the system size in the $y$ direction. Fitting to the shape of the buckling of the $9.4^{\circ}$ boundary produced by MD simulations gives $A_{\mathrm{b}}=0.55 \mathrm{~nm}, \xi=0.72 \mathrm{~nm}$, and $a=0.01$. As in the MD simulations, $L_{y}=4.5 \mathrm{~nm}$, so $m$ must be set to 3 to obtain the correct periodicity in $y$. The system length in the $x$ direction, $L_{x}$, is set to $100 \mathrm{~nm}$.

In addition to the static out-of-plane displacement, the time-independent terms in the stress tensor components are also needed. These have been obtained by fitting to the (approximate) stress tensor components obtained from MD.

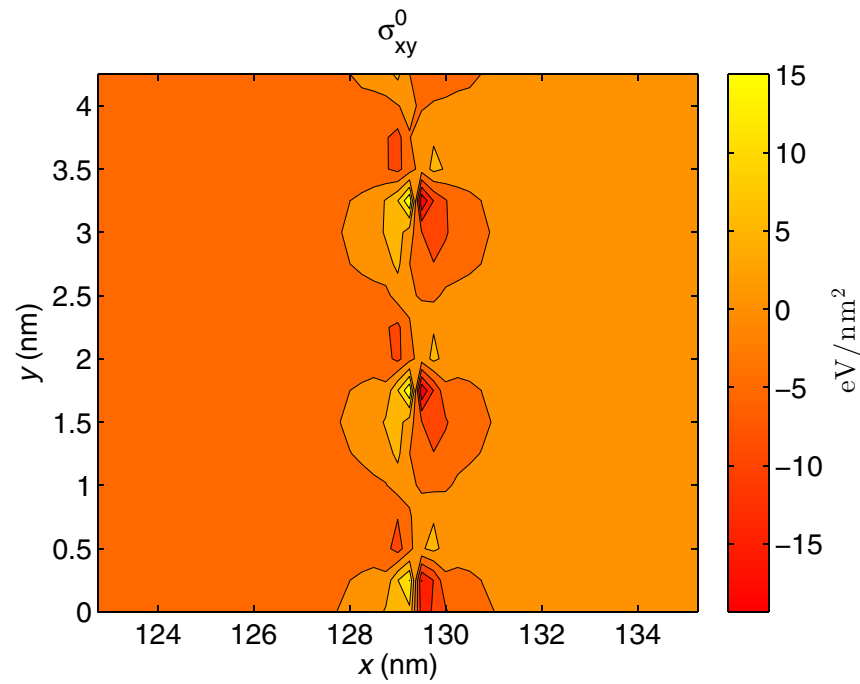

(a)

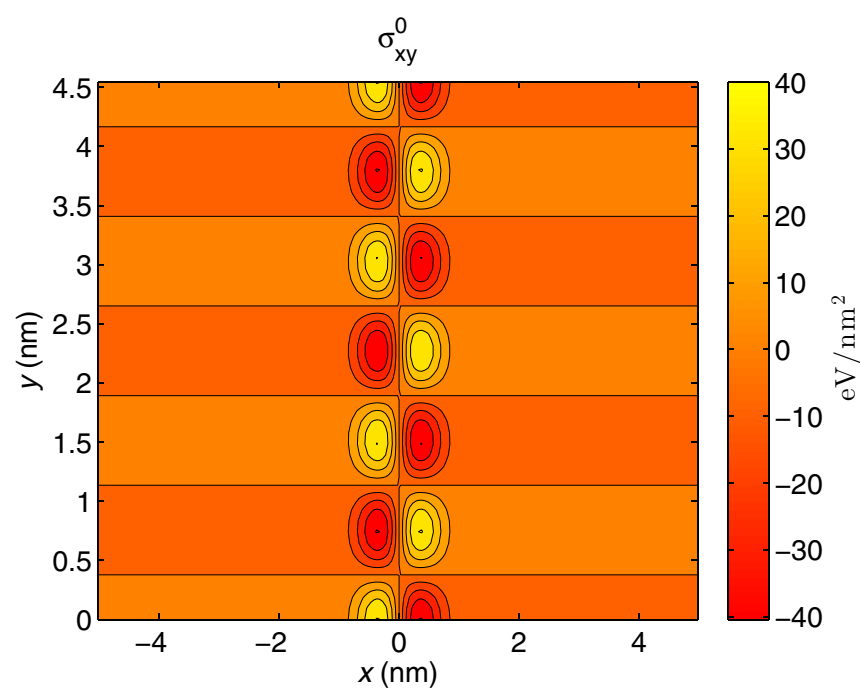

(b)

FIG. 11. (Color online) The stress tensor component $\sigma_{x y}^{0}$ (a) as obtained from MD and (b) as approximated according to Eq. (A17).

Starting with $\sigma_{x x}^{0}$, it is seen that if we set

$$
\sigma_{x x}^{0}=e^{-2 x^{2} / \xi^{2}} \sin \left(\frac{2 \pi m y}{L_{y}}\right)
$$

we obtain a good qualitative correspondence to the MD data (see Fig. 9).

To satisfy the relations between the stress tensor components given by Eq. (A8), we must then set

$$
\begin{gathered}
\sigma_{y y}^{0}=-\left(\frac{L_{y}}{2 \pi m}\right)^{2}\left(-\frac{4}{\xi^{2}}+\frac{16 x^{2}}{\xi^{4}}\right) e^{-2 x^{2} / \xi^{2}} \sin \left(\frac{2 \pi m y}{L_{y}}\right), \\
\sigma_{x y}^{0}=-\left(\frac{L_{y}}{2 \pi m}\right) \frac{4 x}{\xi^{2}} e^{-2 x^{2} / \xi^{2}} \cos \left(\frac{2 \pi m y}{L_{y}}\right) .
\end{gathered}
$$

As can be seen in Figs. 10 and 11, this functional form of the stress tensor components does reproduce the MD result in a qualitative manner. 
In order to compare the results of the continuum mechanical model to those obtained from MD, the transmission coefficient $T_{\mathrm{c}}$ is calculated as

$$
T_{\mathrm{c}}=\left\langle\frac{\Delta y \Delta x \rho \sum_{x_{i}>0} \sum_{y_{j}=0}^{L_{y}^{\mathrm{sc}}}\left(\omega_{x}^{2} u_{1}^{2}\left(x_{i}, y_{j}, t_{n}\right)+\omega_{y}^{2} v_{1}^{2}\left(x_{i}, y_{j}, t_{n}\right)+\omega_{z}^{2} w_{1}^{2}\left(x_{i}, y_{j}, t_{n}\right)\right)}{2 E^{\text {tot }}}\right\rangle,
$$

where $\omega_{x}, \omega_{y}$, and $\omega_{z}$ are the frequencies of vibrations in the $x, y$, and $z$ directions; $x_{i}=i \Delta x$ and $y_{j}=j \Delta y$ indicate a point on the discretization grid; and $t_{n}=n \Delta t$ is the time step. The time average is taken over times after scattering against the static out-of-plane displacement, and the total energy $E^{\text {tot }}$ is given by

$$
E^{\mathrm{tot}}\left(t_{n}\right)=\frac{\Delta y \Delta x \rho}{2} \sum_{x_{i}=-L_{x} / 2}^{L_{x} / 2} \sum_{y_{j}=0}^{L_{y}^{\mathrm{sc}}}\left[\omega_{x}^{2} u_{1}^{2}\left(x_{i}, y_{j}, t_{n}\right)+\omega_{y}^{2} v_{1}^{2}\left(x_{i}, y_{j}, t_{n}\right)+\omega_{z}^{2} w_{1}^{2}\left(x_{i}, y_{j}, t_{n}\right)\right] .
$$

[1] O. V. Yazyev and Y. P. Chen, Nat. Nanotechnol. 9, 755 (2014).

[2] O. V. Yazyev, Solid State Commun. 152, 1431 (2012).

[3] J. Zhang and J. Zhao, Carbon 55, 151 (2013).

[4] G.-H. Lee, R. C. Cooper, S. J. An, S. Lee, A. van der Zande, N. Petrone, A. G. Hammerberg, C. Lee, B. Crawford, W. Oliver, J. W. Kysar, and J. Hone, Science 340, 1073 (2013).

[5] A. Cao and J. Qu, J. Appl. Phys. 112, 043519 (2012).

[6] Z. Fei, A. S. Rodin, W. Gannett, S. Dai, W. Regan, M. Wagner, M. K. Liu, A. S. McLeod, G. Dominguez, M. Thiemens, A. H. C. Neto, F. Keilmann, A. Zettl, R. Hillenbrand, M. M. Fogler, and D. N. Basov, Nat. Nanotechnol. 8, 821 (2013).

[7] Q. Yu, L. A. Jauregui, W. Wu, R. Colby, J. Tian, Z. Su, H. Cao, Z. Liu, D. Pandey, D. Wei, T. F. Chung, P. Peng, N. P. Guisinger, E. A. Stach, J. Bao, S.-S. Pei, and Y. P. Chen, Nat. Mater. 10, 443 (2011).

[8] A. W. Tsen, L. Brown, M. P. Levendorf, F. Ghahari, P. Y. Huang, R. W. Havener, C. S. Ruiz-Vargas, D. A. Muller, P. Kim, and J. Park, Science 336, 1143 (2012).

[9] J. Coraux, A. T. N'Diaye, C. Busse, and T. Michely, Nano Lett. 8, 565 (2008).

[10] J. M. Carlsson, L. M. Ghiringhelli, and A. Fasolino, Phys. Rev. B 84, 165423 (2011).

[11] T.-H. Liu, G. Gajewski, C.-W. Pao, and C.-C. Chang, Carbon 49, 2306 (2011).

[12] C. Gómez-Navarro, J. C. Meyer, R. S. Sundaram, A. Chuvillin, S. Kurasch, M. Burghard, K. Kern, and U. Kaiser, Nano Lett. 10, 1144 (2010).

[13] J. H. Warner, Y. Fan, A. W. Robertson, K. He, E. Yoon, and G. D. Lee, Nano Lett. 13, 4937 (2013).

[14] O. Lehtinen, S. Kurasch, A. V. Krasheninnikov, and U. Kaiser, Nat. Commun. 4, 2089 (2013).

[15] P. Y. Huang, C. S. Ruiz-Vargas, A. M. van der Zande, W. S. Whitney, M. P. Levendorf, J. W. Kevek, S. Garg, J. S. Alden, C. J. Hustedt, Y. Zhu, J. Park, P. L. McEuen, and D. A. Muller, Nature (London) 469, 389 (2011).

[16] K. Kim, Z. Lee, W. Regan, C. Kisielowski, M. F. Crommie, and A. Zettl, ACS Nano 5, 2142 (2011).

[17] J. H. Warner, E. R. Margine, M. Mukai, A. W. Robertson, F. Giustino, and A. I. Kirkland, Science 337, 209 (2012).

[18] A. W. Robertson, C. S. Allen, Y. A. Wu, K. He, J. Olivier, J. Neethling, A. I. Kirkland, and J. H. Warner, Nat. Commun. 3, 1144 (2012).
[19] A. Hashimoto, K. Suenaga, A. Gloter, K. Urita, and S. Iijima, Nature (London) 430, 870 (2004).

[20] A. A. Balandin and D. L. Nika, Mater. Today 15, 266 (2012).

[21] E. Pop, V. Varshney, and A. K. Roy, MRS Bulletin 37, 1273 (2012).

[22] A. Cao and J. Qu, J. Appl. Phys. 111, 053529 (2012).

[23] H.-Y. Cao, H. Xiang, and X.-G. Gong, Solid State Commun. 152, 1807 (2012).

[24] A. Bagri, S.-P. Kim, R. S. Ruoff, and V. B. Shenoy, Nano Lett. 11, 3917 (2011).

[25] Y. Lu and J. Guo, Appl. Phys. Lett. 101, 043112 (2012).

[26] A. Y. Serov, Z.-Y. Ong, and E. Pop, Appl. Phys. Lett. 102, 033104 (2013).

[27] S.-H. Tan, L.-M. Tang, Z.-X. Xie, C.-N. Pan, and K.-Q. Chen, Carbon 65, 181 (2013).

[28] T.-H. Liu, S.-C. Lee, C.-W. Pao, and C.-C. Chang, Carbon 73 , 432 (2014).

[29] J. H. Seol, I. Jo, A. L. Moore, L. Lindsay, Z. H. Aitken, M. T. Pettes, X. Li, Z. Yao, R. Huang, D. Broido, N. Mingo, R. S. Ruoff, and L. Shi, Science 328, 213 (2010).

[30] L. Lindsay, D. A. Broido, and N. Mingo, Phys. Rev. B 82 , 115427 (2010).

[31] E. E. Helgee and A. Isacsson, Phys. Rev. B 90, 045416 (2014).

[32] S. J. Plimpton, J. Comput. Phys. 117, 1 (1995), http://lammps. sandia.gov.

[33] J. Tersoff, Phys. Rev. B 37, 6991 (1988).

[34] J. Tersoff, Phys. Rev. Lett. 61, 2879 (1988).

[35] L. Lindsay and D. A. Broido, Phys. Rev. B 81, 205441 (2010).

[36] W. Humphrey, A. Dalke, and K. Schulten, J. Mol. Graphics 14, 33 (1996).

[37] C. Kimmer, S. Aubry, A. Skye, and P. K. Schelling, Phys. Rev. B 75, 144105 (2007).

[38] J. D. Gale, J. Chem. Soc., Faraday Trans. 93, 629 (1997).

[39] J. D. Gale and A. L. Rohl, Mol. Simulat. 29, 291 (2003).

[40] M. Omini and A. Sparavigna, Phys. Rev. B 61, 6677 (2000).

[41] L. D. Landau and E. M. Lifshitz, Theory of Elasticity, 3rd ed. (Elsevier, Oxford, 1986).

[42] W. H. Press, S. A. Teukolsky, W. T. Vetterling, and B. P. Flannery, Numerical Recipes: The Art of Scientific Computing, 3rd ed. (Cambridge University, New York, 2007). 